\title{
DEVELOPMENT OF IDENTIFICATION SYSTEM FOR CHECK-WEIGHER
}

\author{
TATSUAKI HASHIMOTO*, TOSHITAKA UMEMOTO and ATSUSHI MAEDA \\ Osaka Prefecture University College of Technology, \\ 26-12 Saiwai-cho, Neyagawa, Osaka 572-8572, Japan \\ *umemoto@osaka-pct.ac.jp
}

\begin{abstract}
Our identification system for the natural frequency consisted of control computer, D/AA/D conversion interface boards, motor driver, vibrator and eddy current displacement sensor. The RealTime application interface for Linux was used as the computer OS. First the sensing analog data were converted to the digital data. Then the count values were re-converted to the voltage data by our software. Here we used the enhanced signal compression method using the time stretched pulse signal. In the experiment using the system, the weight of products was confirmed to bear a proportionate relationship to the natural frequency. This indicates that our method is available to identify the natural frequency.
\end{abstract}

Keywords: Signal processing; vibration analysis; realtime Linux.

\section{Introduction}

Both of enough measurement time and noise free condition are necessary accurately to measure the mass. However, it is difficult to make such ideal surroundings in the industrial weighing and sorting systems [1-4]. Such a system is generally called "check-weigher", which is utilized to examine and sort the products in the production lines. The check-weigher have to carry the products from the previous conveyor to the next conveyor and also to exclude some defectives, that is, lightweight or overweight products. In addition, these works must be done at the same time. Namely, it is necessary to analyze not only the measurement method but also the conveyance method in order to develop the check-weigher. The required conditions in the conveyance method are as follows:

1) Stable and reliable weighing results;

2) High conveying speed;

3) Simple and flexible operation mechanism.

This is an Open Access article published by World Scientific Publishing Company. It is distributed under the terms of the Creative Commons Attribution 3.0 (CC-BY) License. Further distribution of this work is permitted, provided the original work is properly cited. 
As the conveyance method with the above conditions, recently, the active belt conveyor is widely adopted in the industry sites. However, since the conventional mass detector is composed of built-in drive, it is impossible to avoid the mechanical noises from the conveyor. The mechanical disturbances, such as natural vibration, motor vibration and the Pully vibration, should be rejected to improve the measurement accuracy. In the current system, these noises are removed by simple moving average, MA, method. This method can completely remove the noises when the interval cycle of the noises synchronizes with the moving average time. However, most of the users need the machines which can quickly measure the objects with various shapes. In order to meet this demand, both of flexibility and speed should be improved in the measurement system. These improvements reduce the measurement time. As a result, all of noises cannot be removed completely. This means that the simple MA method is not appropriate for the practical measurement system. In the present study, therefore, we propose the adoption of adaptive notch filters using spectral analysis method by the LMS algorithm [5-8]. We proposed the system that combines the adaptive notch filters with the simple MA method. For the natural vibration, it is imperative to identify the frequency after the installation of the check-weigher. Impulse response method has been traditionally used for the identification. However, the complete duplication of the impulse signals is intensely difficult. In the present study, therefore, we used the enhanced signal compression method using the time stretched pulse, TSP, signal $[9,10]$. Our identification system for the natural frequency consisted of control computer, D/A-A/D conversion interface boards, motor driver, vibrator and eddy current displacement sensor. The RTAI, RealTime Application Interface for Linux, was used as the computer OS [11]. First the sensing analog data were converted to the digital data. Then the count values were re-converted to the voltage data by our software. In the experiment using the system and the equation described above, the weight of products was confirmed to bear a proportionate relationship to the natural frequency. This indicates that our method is available to identify the natural frequency.

\section{Materials and Methods}

\subsection{Outline of measurement system}

The check-weigher used in this study is the mass measurement system of CSE22L type (Yamato Scale Co., Ltd.), which is shown in Fig. 1. The check-weigher is composed of the carrying and measuring conveyors. The measurement data are accumulated in the check-weigher as the count values. The mass measurement values are shown by the following equation:

$$
M=\left(C-C_{i}\right) \frac{M_{h}}{C_{h}-C_{i}}
$$

Where, $C_{i}$ is the count of standard value, $C_{h}$ the count value at standard weight and $M_{h}$ the standard weight. 


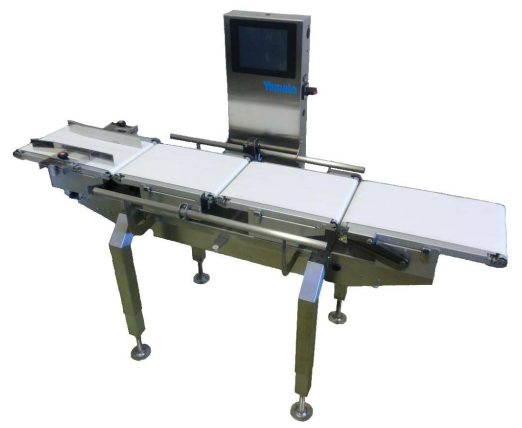

Fig. 1. Our Check-weigher.

\subsection{Noise sources in equipment}

As described in the introduction, the active belt conveyors possess the mechanical noises, that is, the natural vibration in the measurement conveyor, the vibration of motor, and the belt pulley. The noise frequency from the motor and the belt pulley can be calculated from the conveyor speed. If the conveyor speed is set at $v_{c}$, the rotation of the belt pulley is defined as follows:

$$
f_{p}=\frac{v_{c}}{\pi d_{p}}
$$

where, $p_{d}$ is the diameter of the belt pulley.

$f_{m}$ can be calculated by the ratio of the number of the motor's teeth, $M_{m}$, and the pulley's teeth, $M_{p}$.

$$
f_{m}=\frac{M_{m}}{M_{p}} f_{p}
$$

Therefore, the elements of the frequencies are estimated by using these equations when the users fix the conveyor speed. Then, the natural vibration in the equipment can be determined in the design stage. In this study, we have developed a system for determining the natural vibration in the equipment.

\section{Signal Compression Method}

The signal compression method can be summarized with the help of Fig. 2. The "Time Stretched Pulse signal(TPS signal)" in the figure is a special computergenerated test signal whose power spectrum is flat in a specified frequency range. A computational procedure for this signal is as follows: a frequency domain signal is defined first, so as to have a flat power spectrum and special phase characteristics. The phase angle of the complex frequency function is defined to be proportional to the frequency squared. Since the delay time of a sinusoidal or a narrow-band signal is proportional to a derivative of the phase function with respect to frequency the delay time of each frequency component of the test signal is proportional to the frequency itself. Therefore, TSP signal is a rather long time signal as can be seen in 


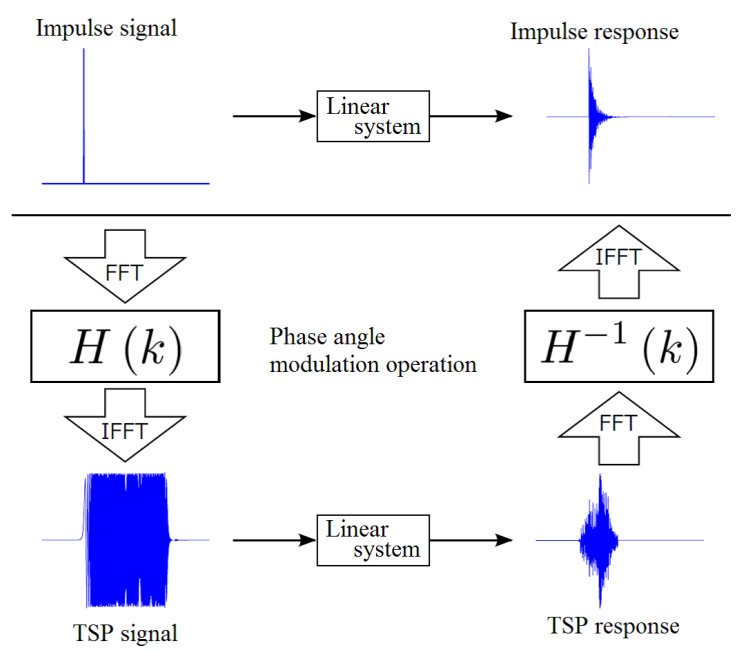

Fig. 2. The principle diagram of the Signal Compression Method.

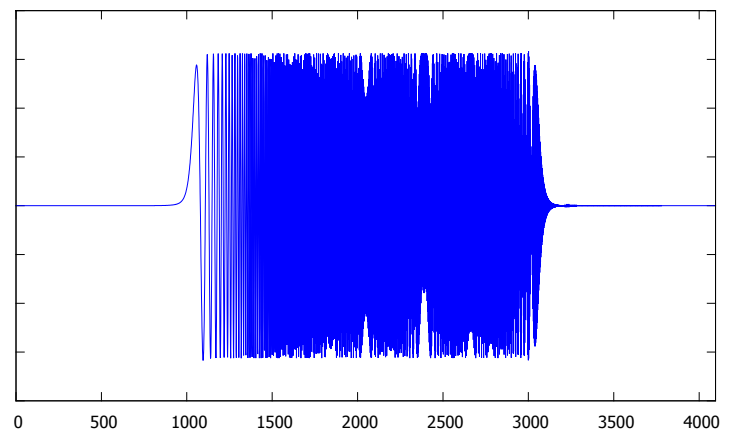

Fig. 3. Time Stretched Pulse signal.

Figure 3, although the high-frequency components of this figure are attenuated by the amplifier and filter characteristics used. High-frequency components come first and low-frequency components next because of a minus sign in an expression of a delay time (actually it is an advance time). The generation of the test signal can be explained conceptually as follows: if an impulse signal located at the time origin is Fourier transformed, the complex frequency function has a flat spectrum and the phase angle is constantly zero. By multiplying the complex frequency function by

$$
H(\omega)=\exp \left(j \alpha \omega^{2}\right) .
$$

The desired frequency function is obtained. The expanded test signal is derived by an inverse Fourier transformation. This means an impulse signal is expanded to a long duration signal by a Fourier transformation(FFT), a numerical phase shift filter $[H(\omega)]$, and an inverse Fourier transformation(IFFT). This also means the 


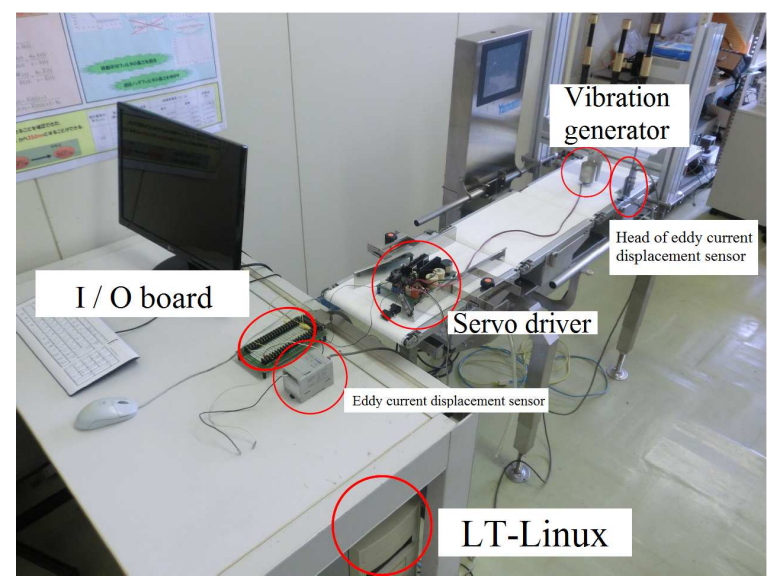

Fig. 4. Our system.

test signal can be compressed to an impulsive signal by an inverse process of the above mentioned, that is, FFT, $H^{-1}(\omega)$, and IFFT. The inverse filter is given as

$$
H^{-1}(\omega)=\exp \left(-j \alpha \omega^{2}\right)
$$

This inverse process is herein referred to as a "signal compression." The expanded signal is an input to a linear system which we desired to investigate. The response of the linear system to the expanded test signal is measured and then it is compressed numerically. These procedures are summarized as

(A) Impulse signal $\stackrel{\text { Expansion }}{\longrightarrow}$ Expanded signal $\stackrel{\text { Linear system }}{\longrightarrow}$ Measured response $\stackrel{\text { Compression }}{\longrightarrow}$ Compressed response.

Three operations of the signal expansion, signal compression, and obtaining response by the linear system are all linear, so that they can be interchanged without any change in the final result. This implies that the next sequence gives the same response as the above.

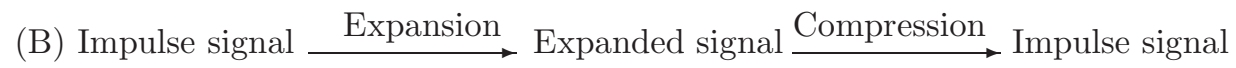

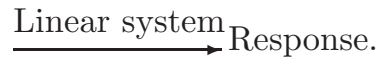

Obviously the first two steps can be omitted, so the next sequence is obtained.

(C) Impulse signal $\underset{\text { Linear system }}{\longrightarrow}$ Response.

This is just an ordinary impulse response measurement. Now the impulse response of a liner system can be obtained by compressing the response of the system to TSP signal as shown schematically in Fig. 3. An advantage of using the complex procedure of (A) instead of $(\mathrm{C})$ is the large power of the expanded signal. Since in all practical experiments, signal amplitude is inherently limited, the time expansion is useful way of increasing signal power. The filters of Eqs. (4) and (5) cannot be 


\begin{tabular}{|c|c|}
\hline Equipment & Detail \\
\hline Computer & Pentium 4 (2.2GHz), 512MB RAM \\
\hline OS & Vine Linux 4.2 (Kernel:Ver.2.6.32.2) \\
& RTAI Ver.3.8.1 is applied. \\
\hline Interface Board & AD12-16 (PCI) E(CONTEC CO., LTD:) \\
\hline Motor driver & SMCM2-AI(Servo Land Co., Ltd.) \\
\hline Vibration generator & VCM26-02R \\
& (Showa Electric Wire \& Cable Co., Ltd. ) \\
\hline Eddy current displacement sensor & EX-210 + EX-422(Keyence Corporation:) \\
\hline
\end{tabular}

realized physically but it is not a problem because they are used only in a computer for known numerical data.

\subsection{Identification apparatus}

Figure 4 and Table 2 show the external appearance and the components of our system, respectively. The identification apparatus comprised control PC, D/A-A/D converter interface boards, power amplifier driver, vibration generator, and eddycurrent displacement sensor. In detail, the $\mathrm{D} / \mathrm{A}$ and $\mathrm{A} / \mathrm{D}$ converter interface boards were added to the general PC with Realtime Linux OS, RTAI [6]. The PC controlled the input to the motor driver and the output from the sensor. After the amplification to the amplitude of about $15 \mathrm{~V}$, the output signal was entered into the vibration generator. The tiny response vibration was measured using the eddy-current displacement sensor. Original software converted the output analog data to the voltage values. The generated CSV files were processed by the numerical software such as Matlab. It is worthy to note that the height of the vibration generator can be arbitrarily adjusted to meet changes of the measurement objects.

\section{Experimental}

First the check-weigher was started with no objects on the measurement conveyor to calibrate the zero point. Next, DC $3 \mathrm{~V}$ was applied to the vibration generator. This is because the constantly-contiguous between the object and the conveyor realizes the input of the correct TSP signal. Here the input signal voltage is not equal to the input voltage to the vibration generator, which is activated through the power amplifier driver. Thus, the weight of the object was measured with DC voltage. The response of the input TSP signal was received after this process. To increase the $\mathrm{S} / \mathrm{N}$ ratio, synchronous addition was done a hundred times. The sampling frequency was constant at $300 \mathrm{~Hz}$. Figure 3 shows the TSP signal used in the experiment.

\subsection{Natural frequency identification}

The natural frequency was initially measured under the minimum weight of $300 \mathrm{~g}$. The input signal and the corresponding check-weigher response were shown in 


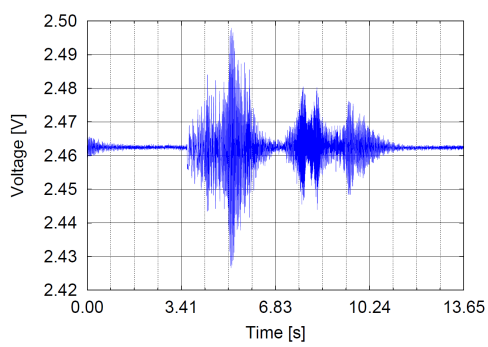

Fig. 5. TSP response (object weight: $300 \mathrm{~g}$ ).

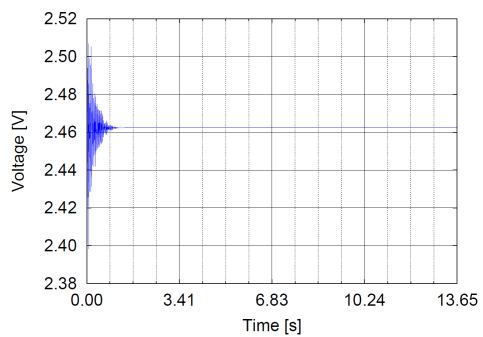

Fig. 7. Impulse response without noise (object weight: $300 \mathrm{~g}$ ).

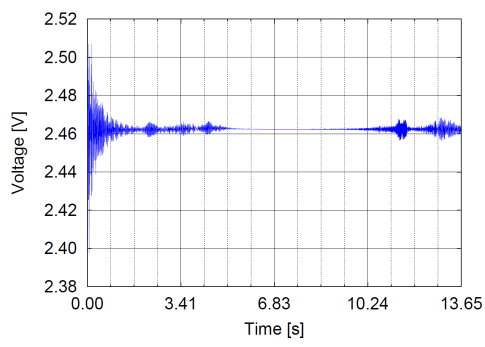

Fig. 6. Impulse response (object weight: $300 \mathrm{~g})$.

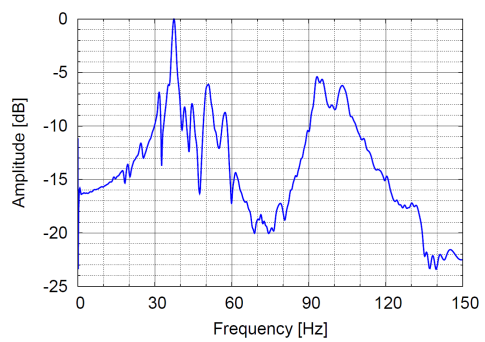

Fig. 8. Amplitude characteristics (object weight: $300 \mathrm{~g})$.

\begin{tabular}{|c|c|c|}
\hline Mass [g] & Natural vibration $[\mathrm{Hz}]$ & Filter length $M$ \\
\hline 300 & 37.50 & 13 \\
\hline 600 & 36.47 & 13 \\
\hline 900 & 35.60 & 14 \\
\hline 1200 & 34.06 & 14 \\
\hline 1500 & 32.96 & 15 \\
\hline
\end{tabular}

Figs. 3 and 5, respectively. Here the TSP signal contains the energy between 2.5 and 11 sec. In the other time, only the noises were observed. Based on these facts, the inverse data were convoluted using the hamming window, as shown in Fig. 6. In our system, the check-weigher possessed the damped vibration with the time constant of $1.3 \mathrm{sec}$ about the impulse input. After $1.3 \mathrm{sec}$, therefore, the data in Fig. 6 were ignorable. Figure 7 shows the recalculation results using the hamming window. The amplitude characteristics were evaluated by the Fourier transform (see Fig. 8). As shown in Fig. 8, the amplitude reached a maximum at $37.5 \mathrm{~Hz}$. Since the values were smaller than $-5 \mathrm{~dB}$ at other frequency, the natural frequency under the object weight of $300 \mathrm{~g}$ was concluded to be $37.5 \mathrm{~Hz}$. 


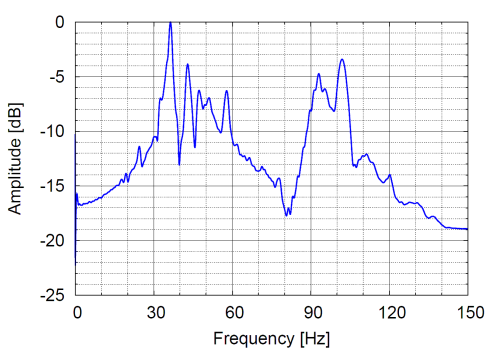

Fig. 9. Amplitude characteristics (object weight: $600 \mathrm{~g}$ ).

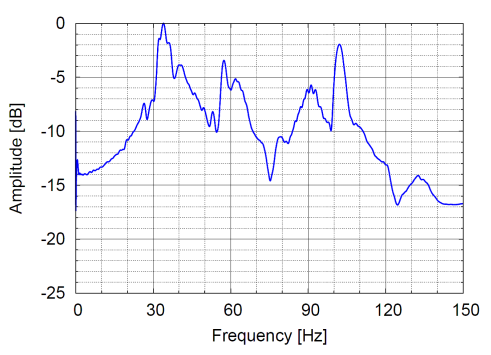

Fig. 11. Amplitude characteristics (object weight: $1200 \mathrm{~g})$.

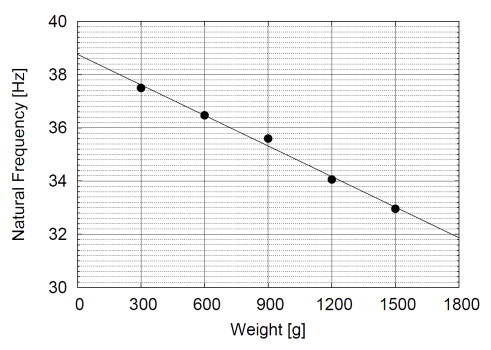

Fig. 13. Correlation between object weight and natural frequency.

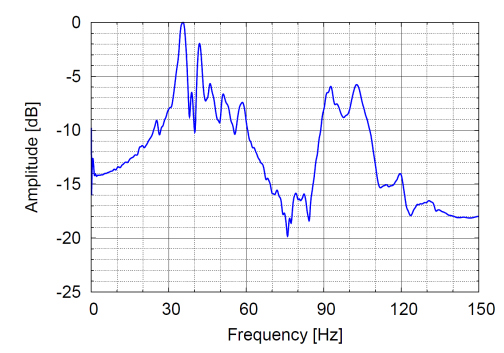

Fig. 10. Amplitude characteristics (object weight: $900 \mathrm{~g}$ ).

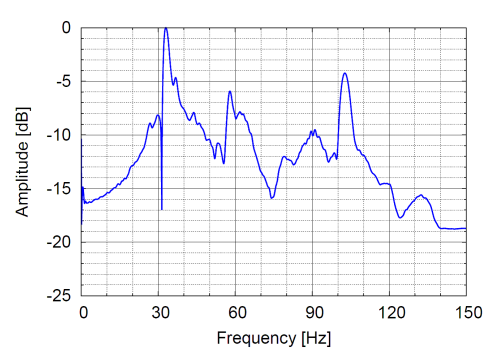

Fig. 12. Amplitude characteristics (object weight: $1500 \mathrm{~g})$.

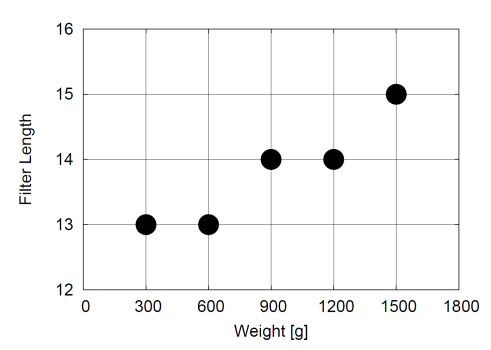

Fig. 14. Correlation between object weight and filter length.

\subsection{Filter length optimization}

The equation for the optimum filter length can be derived from the relation between the object weight and the natural frequency. In the present study, therefore, the natural frequency was measured with the weight change from $600 \mathrm{~g}$ to $1500 \mathrm{~g}$ at the interval of $300 \mathrm{~g}$. The results are shown in Figs. 9, 10, 11, and 12. The natural frequency linearly decreased with the increase of the object weight (see Fig. 13 and 
Table 5.1). The correlation equation, Eq. (6), was obtained by the method of least squares. The natural frequency was concluded to be $38.8 \mathrm{~Hz}$ for the check-weigher with no loads:

$$
f_{n}(x)=-0.00383 x+38.7644 .
$$

The filter length, $M$, can be estimated by the sampling period of the check-weigher, $T$, and the disturbance period, $d$, as follows:

$$
M=\frac{d}{T}
$$

where the disturbance period, $d$, is defined by the reciprocal factor of the natural frequency as shown in Eq. (8). In addition, the sampling frequency, fs, is the inverse of the sampling period, T (see Eq. (9)). As the result, the conversion equation of Eq. (7) is Eq. (10):

$$
\begin{gathered}
d=\frac{1}{f_{n}} \\
f_{s}=\frac{1}{T} \\
M=\frac{f_{s}}{f_{n}} .
\end{gathered}
$$

Since the sampling period of the check-weigher was $2 \mathrm{msec}$, the sampling frequency, $f_{s}$, was $500 \mathrm{~Hz}$. In the present study, therefore, the filter length, $M$, can be shown by Eq. (11):

$$
M=\frac{500}{-0.00383 x+38.7644}
$$

Table 1 shows the triadic relationship between the object weight, the natural frequency and the filter length. It is found that the filter length should be increased by 1 every $600 \mathrm{~g}$ (see Fig. 14).

\section{Conclusion}

The check-weigher is now attracting attention from various industrial fields. However, the current models possess the serious technical problem associated with three types of mechanical noises. This is because the disturbance vibration is overlapped with the measurement waveform. In mass measurement systems, the moving average, MA, filter is applied to remove such noises. Here it is necessary to accurately know the frequency of the disturbance vibration. For the motor vibration and the belt pulley vibration, the frequency can be decided by setting the driving condition. In contrast, the natural vibration should be measured for each check-weigher. In the present study, we prepared the natural frequency identification system using the signal compression method proposed by Aoshima. 
The signal compression method is derived from the impulse response approach. Namely, first, the phase of the impulse signal is changed with the square of the frequency. Next, the elongation in the time axis increases the energy. As the result, we can obtain the TSP signal with higher $\mathrm{S} / \mathrm{N}$ ratio in comparison with the impulse signal. In the present study, the signal compression method was demonstrated to be effective for the vibration analysis in addition to the acoustic analysis. The natural frequency of the check-weigher changes with the weight of the measurement object. Therefore, the equation for the optimum filter length was derived by the measurement of the object weight and the natural frequency. The experimental results indicated that the linear approximation could be applied to the relation between them. It was also found that the filter length should be increased by 1 every $600 \mathrm{~g}$.

\section{References}

1. Ono T., "Basic points of dynamic mass measurement," Proc. SICE Annual Conference, Iwate, 1999, pp.43-44.

2. Ono T., "Dynamic Mass Measurement," Japan Society for Design Engineering, Vol.38, No.10, pp.489-495, 2003.

3. Kurosu S. et al., "Continuous Mass Measurement on Conveyor Belt," Institute of Electrical Engineers of Japan C, Vol.126, No.2, pp.264-269, 2006 [in Japanese].

4. Kurosu S. et al., "Improved Continuous Weighing by Multi-stage Conveyor Belt Scale," Society of Instrument and Control Engineers, Vol.40, No.12, pp.1205-1210, 2004 [in Japanese].

5. Umemoto T. and Aoshima H., "The Adaptive Spectrum Analysis for Transcription," Society of Instrument and Control Engineers, Vol.28, No.5, pp.619-625, 1992 [in Japanese].

6. Umemoto T. and Aoshima H., "Selection of Step Size Parameter of the Adaptive Filter for Spectrum Analysis," Society of Instrument and Control Engineers, Vol.28, No.10, pp.1257-1262, 1992 [in Japanese].

7. Umemoto T. and Aoshima H., "Adaptive DFT and its Application," Society of Instrument and Control Engineers, Vol.30, No.8, pp.959-965, 1994 [in Japanese].

8. Umemoto T. and SasamotoY., "Frequency Analysis Method using the Adaptive Algorithm and Application to Dynamic Mass Measurement of Mass and Weight," Japan Society of Mechanical Engineers, Vol.74, No.8, pp30-36, 2006 [in Japanese].

9. AOSHIMA N. Computer-generated pulse signal applied for sound measurement. J. Acoust. Soc. Am., Vol. 69, pp. 1484.1488, 1981.

10. SUZUKI Y. An optimum computer-generated pulse signal suitable for the measurement of very long impulse responses. J. Acoust. Soc. Am., Vol. 97, No. 2, pp.1119.1123, 1995.

11. Rtai - official websize. https://www.rtai.org/. 